\title{
Effect of questions used by psychiatrists on therapeutic alliance and adherence
}

Laura Thompson, Christine Howes and Rose McCabe

\section{Background}

Psychiatrists' questions are the mechanism for achieving clinical objectives and managing the formation of a therapeutic alliance - consistently associated with patient adherence. No research has examined the nature of this relationship and the different practices used in psychiatry. Questions are typically defined in binary terms (e.g. 'open' $v$. 'closed') that may have limited application in practice.

\section{Aims}

To undertake a detailed examination of the types of questions psychiatrists ask patients and explore their association with the therapeutic alliance and patient adherence.

\section{Method}

A coding protocol was developed to classify questions from 134 out-patient consultations, predominantly by syntactic form. Bivariate correlations with measures of patient adherence and the therapeutic alliance (psychiatrist-rated) were examined and assessed using generalised estimating equations, adjusting for patient symptoms, psychiatrist identity and amount of speech.

\section{Results}

Psychiatrists used only four of ten question types regularly: yes/no auxiliary questions, 'wh-' questions, declarative questions and tag questions. Only declarative questions predicted better adherence and perceptions of the therapeutic relationship. Conversely, 'wh-' questions associated with positive symptoms - predicted poorer perceptions of the therapeutic relationship. Declarative questions were frequently used to propose an understanding of patients' experiences, in particular their emotional salience for the patient.

\section{Conclusions}

A refined defining of questioning practices is necessary to improve communication in psychiatry. The use of declarative questions may enhance alliance and adherence, or index their manifestation in talk, e.g. better mutual understanding. The function of 'so'-prefaced declaratives, also used in psychotherapy, is more nuanced than negatively connotated 'leading' questions. Hearable as displays of empathy, they attend closely to patient experience, while balancing the tasks of assessment and treatment.

\section{Declaration of interest}

None.

\section{Copyright and usage}

(c) The Royal College of Psychiatrists 2016.
Psychiatry is inconceivable without clinician questions. They are the mechanism for achieving clinical objectives: history taking, reviewing symptoms and deducing diagnostic hypotheses. Questioning also manages the formation of a therapeutic alliance, the benefits of which include concordant treatment decisions and patient adherence. ${ }^{1}$ Developing evidence-based interviewing techniques to improve these outcomes is crucial, particularly in the case of schizophrenia where psychotic symptoms may problematise interaction. ${ }^{2}$ A conceptual issue hinders this in practice - there is no definitive model of 'good' communication. ${ }^{3}$ Instead, it is viewed more generically through the ideology of 'patient-centredness', i.e. accounting for the patient's psychosocial context, preference and experience. Although questions are the mode of eliciting this experience, advice in psychiatry textbooks is often limited and generalised, e.g. 'in general try to use open questions rather than leading questions or closed questions. ${ }^{4}$ In practice, 'open' and 'closed' categories encompass numerous linguistic question types, each of which may have different interactional consequences. ${ }^{5}$ No research to date has examined the actual questions - by means of a sensitive, utilitarian classification - that psychiatrists deploy in clinical encounters and how they are linked to the therapeutic alliance and treatment adherence. To specify training and improve these outcomes, we must first explore two research questions, the aims of this study: first, what types of questions do psychiatrists ask patients in routine consultations, and second, do particular question types predict better therapeutic alliances and treatment adherence?

\section{Method}

Data were drawn from a UK Medical Research Council study examining clinical interaction in psychosis, ${ }^{6}$ collected between 2006 and 2008. Thirty-six psychiatrists from out-patient and assertive outreach clinics across three centres in England (one urban, one semi-urban and one rural) were randomly selected, of whom 31 consented to participate (86\%). Their patients meeting DSM-IV criteria for schizophrenia or schizoaffective disorder were also invited to participate. ${ }^{7}$ Of 579 eligible consecutive attenders, 188 persons did not attend their appointment, 42 were not approached for clinical reasons (deemed too unwell) or logistical reasons (overlapping appointments) and 211 declined to take part. Written informed consent was obtained from 138 (40\%) of those invited, following which their consultations were audiovisually recorded. Four encounters were excluded owing to inadequate recording quality. Verbal dialogue was transcribed verbatim; the final set of 134 transcripts formed our data-set.

\section{Question coding}

A standardised protocol (online Fig. DS1) was developed and piloted collaboratively by a team with experience in linguistics (C.H.) and psychiatric communication (L.T. and R.M.). Regular meetings facilitated the refinement of the protocol, applied by all team members to transcripts of video-recorded consultations in an iterative piloting process. The resulting coding scheme 
allowed an exhaustive classification of questions within each transcript. Question taxonomies that move beyond an 'open' v. 'closed' conceptualisation vary according to the accepted meaning of the question itself: broadly, syntactically (by form), semantically (by meaning) or pragmatically (by function). ${ }^{8}$ Based on examination of the transcripts, our approach used a combination of these classifications to identify and distinguish all items of interest. Where possible, questions were identified by their syntactic form. However, although there are two types of sentence forms that constitute syntactic questions in English - starting the sentence with a 'wh-' word, and swapping the order of the sentence's subject and auxiliary verb (subject-auxiliary inversion), ${ }^{9}$ these are by no means the only ways that questions may be asked. For example, specific lexical items may be commonly used as and taken to be questions (e.g. 'Pardon?'), ${ }^{10}$ and sentences that are syntactically identical in form to non-interrogatives may be used and identified as questions by their rising (questioning) intonation. The classification sought to identify all of these question types.

\section{Question categories}

The complete coding protocol was constructed to be usable without specific knowledge of linguistics. Each candidate utterance was tested against a hierarchy of yes/no format questions, formulated to be as simple as possible (Fig. DS1). A process of sequential elimination thereby identifies the linguistic type of any question, and this process is repeated on the utterance until no further questions are identified. There are ten possible categories:

(a) Yes/no questions: 'Do you ever feel someone is controlling your mind?'

(b) 'Wh-' questions: 'Where was that done?'

(c) Declarative questions: 'So you feel a bit anxious?'

(d) Tag questions: 'You're on $10 \mathrm{mg}$ of olanzapine, aren't you?'

(e) Lexical tags: 'I'll write a letter to your GP, okay?'

(f) Incomplete questions: 'Your keyworker is?'

(g) Alternative questions: 'Do you feel better having stopped it, or worse?'

(h) Check questions: 'Yeah?'

(i) 'Wh-' in situ: 'He did what?'

(j) Open class repair initiators: 'Pardon?'

\section{Yes/no questions}

Yes/no questions are one of the class of 'closed' questions because their expected answer is 'yes' or 'no.' ${ }^{11}$ They are syntactically identifiable with an auxiliary verb in the first position of the sentence, followed by the subject. Auxiliary verbs often express distinctions of tense, aspect or mood and include the verbs do, can, will, have, did: 'Did you really believe it at the time?', 'Have you asked your GP about that?', 'Will you think about reducing your depot?'

\section{'Wh-' questions}

'Wh-' questions have a question word in the first position: who, what, when, why or how. Accordingly, they elicit information on a state of affairs or the property of an event. 'Wh-' questions are considered to be open questions because they do not project a specific response: 'How does that make you feel?', 'What do you mean?', 'Who is your keyworker?'

\section{Declarative questions}

Declarative questions have the syntax of a declarative sentence. ${ }^{11} \mathrm{~A}$ rising intonational contour is likely to index recognition of declaratives as questions, ${ }^{12,13}$ i.e. requiring (dis)confirmation from the patient. Questioning intonation was annotated in the transcripts, so we included all declarative sentences followed by a question mark. Coders also looked to the next turn (the patient response) to see whether the sentence had indeed been understood as a question. Declarative questions are considered to be 'closed' questions, ${ }^{14}$ because they invite yes/no type responses: 'You feel happy about that?', 'You're still on the same medication?', 'Sleeping okay?

\section{Tag questions}

A tag question transforms a declarative statement or imperative into a question by adding an interrogative fragment (the tag), i.e. an auxiliary verb followed by a pronoun: isn't it, would he, do you. Like yes/no questions and declaratives, tag questions can be seen as inviting confirmation or disconfirmation from the patient, thus are another class of 'closed' question: 'You're on $20 \mathrm{mg}$ now, aren't you?', 'You were thinking about working in old people's homes, weren't you?'

\section{Lexical tags}

Lexical tags also invite confirmation or disconfirmation by adding an interrogative fragment to a statement. A list of words that could act as lexical tags, such as right, okay, yeah, you know, was provided to coders. Lexical tags transcribed with a question mark were included, for example: 'We can increase the dose, okay?', 'Sometimes it can take a bit of adjusting to, you know?'

\section{Incomplete questions}

Grammatically incomplete sentences that invited a candidate completion by the patient were coded as incomplete questions. They may be initially formulated as another syntactic structure, e.g. a declarative or alternative question, but invite - through questioning intonation - the patient to complete the missing component: 'You've got a job, or?', 'You take that at night, or?'

\section{Alternative questions}

Like yes/no questions, alternative questions have an auxiliary verb in the first position, but present two or more possible answers that the patient may choose, e.g. 'Do you prefer morning or afternoon?', 'Are you taking that regularly or just when you need it?'

\section{Check questions}

Check questions are synonymous in form with lexical tags, but follow a statement by the patient:

\section{Patient: 'I'd be happy with that.'}

Doctor: 'Yeah?'

\section{'Wh-' in situ}

'Wh-' in situ questions are formed using 'wh-' words, but as a replacement for content words instead of at the beginning of the sentence:

\section{'John went to the zoo'}

'John went where?' (cf. Where did John go?)

Examples are: 'You did that when? 'He said what?' 


\section{Open class repair initiators}

Psychiatrists may draw attention to a problem of hearing or understanding the patient's prior turn using questions that are open class repair initiators, i.e. they 'flag' trouble with the patient's prior turn of talk, but leave open the nature of the problem: ${ }^{10}$ examples are 'Pardon?', 'Sorry?', 'What?', 'Huh?'

\section{Application of the protocol}

A software suite designed for the annotation of language data, Dexter Coder, was used to apply the protocol. ${ }^{15}$ Four raters performed the coding independently. Transcripts consisted of verbal dialogue, therefore assigned question codes were based only on surface syntax, intonational cues and patient responses. Interrater reliability was found to be good for all question types using Cohen's kappa, which ranged from 0.76 to 0.89 .

\section{Measures and outcomes}

Symptoms were assessed immediately post-consultation, and psychiatrists rated their view of the therapeutic relationship for each patient. Patient treatment adherence was assessed by psychiatrists in a follow-up interview, 6 months after the consultation.

\section{Symptoms}

Symptoms were assessed as a potential confounding factor in interviews by researchers not involved in the patient's treatment. The Positive and Negative Syndrome Scale (PANSS) was employed in which 30 items rated 1-7 assess positive, negative and general symptoms, with higher scores denoting greater severity. ${ }^{16}$ Positive symptoms indicate a change in the patient's behaviour or thoughts, e.g. delusions or sensory hallucinations. Negative symptoms represent a reduction in functioning, including blunted affect, emotional withdrawal and alogia. Subscale scores for positive and negative symptoms range from 7 (absent) to 49 (extreme); scores for general symptoms such as anxiety range from 16 (absent) to 112 (extreme). Interrater reliability using audiovisually recorded interviews was good (Cohen's $\kappa=0.75$ ).

\section{Therapeutic alliance}

Psychiatrist perceptions of the therapeutic alliance were assessed post-consultation using the Helping Alliance Scale. ${ }^{17}$ Five items were rated 1-10 on various interpersonal variables including mutual understanding about providing necessary treatment and rapport with the patient. Ratings for individual items were combined to create a single value. A lower score represented a poorer therapeutic relationship.

\section{Adherence to treatment}

Mean percentage adherence, grouped in clusters as recommended by Velligan $e t a l,{ }^{18}$ was assessed 6 months after the consultation by the patient's psychiatrist. Psychiatrists used collateral information to assess adherence in $50 \%$ of cases. In $56 \%$ of these cases this was attendance for depot injection, supervised drug intake or blood tests. In $44 \%$ this was from others involved in the patient's care (e.g. pharmacist, general practitioner, family member).

Adherence to treatment in general (i.e. the percentage of occasions that scheduled appointments were kept and non-medication recommendations were followed) and adherence to medication (i.e. the percentage of medication taken) were rated separately on a three-point scale: $>75 \%$ (rated 1), $25-75 \%$ (rated 2) and $<25 \%$ (rated 3). ${ }^{19}$ The two scores were summed to yield a total adherence score ranging from 2 to 6 , with a lower score indicating better adherence.

\section{Statistical analysis}

Statistical analyses were conducted using SPSS version 18.0. ${ }^{20}$ Descriptive data, including frequencies and means, on question types were retrieved to address our first research question. To explore our second research question, bivariate correlations between each question type and the primary outcomes were performed, establishing significant associations to motivate further analysis. Initially, correlations with symptoms, a potential confounder, were explored. Coefficients were then obtained for adherence and the therapeutic alliance. The associations between question types (the independent predictors) and the primary outcomes (the dependent variables: adherence, the therapeutic alliance) were further assessed using generalised estimating equations (GEE). A GEE analysis was used to account for within-individual correlations. ${ }^{21,22}$ The unit of analysis was the consultation. As each psychiatrist was involved in consultations with several patients, psychiatrist identity was entered as a within-individual factor; this militates against the possibility that personal interviewing style might exert a disproportionate effect on the results. In addition, as the correlations showed that symptoms and question types were not independent, the three symptom scales were also entered as within-individual factors.

\section{Results}

Questions were coded in 134 consultations involving 30 psychiatrists; of these clinicians, $63 \%$ were men and $72 \%$ were of White ethnic origin. Consultations lasted a mean length of $17.2 \mathrm{~min}$ (s.d. =9.1). A total of 114 patients were recruited from out-patient clinics and 24 from assertive outreach clinics. Table 1 displays their sociodemographic and clinical characteristics.

\section{Types of questions asked}

Psychiatrists asked patients a total of 7570 questions across 134 consultations with a mean of 51.7 (s.d.=32.1) questions per consultation. Table 2 lists the mean frequencies of the specific question types in descending order. As length and density of doctor utterances varied between consultations, means were also normalised by calculating values per 1000 words; this controlled

Table 1 Patient sociodemographic and clinical characteristics

\begin{tabular}{lc} 
Sociodemographic variables, $n(\%)^{\text {a }}$ \\
Gender \\
$\quad$ Male & \\
Female & $87(63)$ \\
Ethnicity, White & $47(37)$ \\
Employment & $100(72)$ \\
$\quad$ Unemployed & \\
Employed/student & $86(62)$ \\
$\quad$ Voluntary & $30(22)$ \\
Retired & $10(7)$ \\
Clinical variables, mean (s.d.) & $8(6)$ \\
Age, years & \\
Years in contact with psychiatric services & $42.2(11.5)$ \\
Number of admissions & $15.6(11.6)$ \\
Number of involuntary admissions & $3.4(3.4)$ \\
Symptoms (PANSS score) & $1.8(2.6)$ \\
$\quad$ Total score & \\
Positive & $54.4(18.6)$ \\
$\quad$ Negative & $13.1(5.9)$ \\
$\quad$ General & $12.5(5.8)$ \\
PANSS, Positive and Negative Syndrome Scale. & $28.8(9.6)$ \\
a. Total sample $n=138$, data analysed for $n=134$. & \\
\hline
\end{tabular}




\begin{tabular}{|c|c|c|c|c|c|}
\hline & Total & Mean (s.d.) & Range & Mean per 1000 words (s.d.) & Range \\
\hline All questions & 7570 & $51.7(32.1)$ & 165 & $35.0(16)$ & 93 \\
\hline Yes/no questions & 2362 & $16.5(12.2)$ & 57 & $12.0(6)$ & 30 \\
\hline 'Wh-' questions & 1700 & $12.7(10.4)$ & 63 & $8.5(4.8)$ & 23 \\
\hline Declarative questions & 1648 & $11.0(8.3)$ & 47 & $9.0(8)$ & 40 \\
\hline Tag questions & 842 & $3.9(4.5)$ & 25 & $2.3(2.1)$ & 11 \\
\hline Lexical tags & 496 & $3.7(5.2)$ & 29 & $2.0(2.2)$ & 11 \\
\hline Incomplete questions & 196 & $1.5(1.7)$ & 8 & $1.1(1.8)$ & 12 \\
\hline Alternative questions & 159 & $1.2(1.5)$ & 10 & $0.8(1.2)$ & 9 \\
\hline Check questions & 85 & $0.6(1.4)$ & 7 & $0.4(1.2)$ & 6 \\
\hline 'Wh-' in situ & 47 & $0.4(1)$ & 10 & $0.2(0.6)$ & 5 \\
\hline Open class repair initiators & 35 & $0.3(0.7)$ & 4 & $0.2(0.6)$ & 4 \\
\hline
\end{tabular}

for the possibility that higher question frequencies were due to some psychiatrists talking more. Most frequently psychiatrists asked patients yes/no questions (mean $=16.5)$, followed by 'wh-' questions $($ mean $=12.7)$, declarative questions $($ mean $=11.0)$ and tag questions $($ mean $=3.9)$. Given the relatively low raw frequency of the remaining linguistic types, only these four categories were sufficiently frequent to include in statistical analyses exploring associations with the therapeutic alliance and adherence.

\section{Correlations with outcomes}

Bivariate associations between outcomes and the four most frequent question formats were examined using Spearman correlations. Correlation coefficients and values of significance for each measure are reported below. Statistically significant findings (at the $P<0.05$ level) are described.

\section{Symptoms}

Because symptom severity in schizophrenia can affect communication, we explored correlations between each question type and the three PANSS subscales (positive, negative, general). Yes/no questions were positively correlated with negative symptoms and 'wh-' questions were positively correlated with positive symptoms (Table 3). Declarative and tag questions were not associated with any symptom subtype.

\section{Therapeutic alliance}

Correlations between the therapeutic alliance as measured by Helping Alliance Scale scores and question type are shown in Table 3. Only declarative questions were associated with better clinician perceptions of the therapeutic alliance $(P<0.001)$.

\section{Adherence}

Only psychiatrists' use of declarative questions was negatively correlated with the adherence scale (Table 3), i.e. greater use of declarative questions from the psychiatrist was associated with higher patient adherence at follow-up $(P<0.05)$.

\section{Between-psychiatrist variation}

Given the significant correlations with both therapeutic alliance and adherence, we examined individual variation in psychiatrists' use of declarative questions to consider how clinician identity may influence these outcomes. The number of consultations and mean number of declarative questions, normalised per 1000 words, for each psychiatrist are listed in online Table DS1, as well as the range (the minimum and maximum number of declarative questions). There was great variation in the number of declaratives used, with means varying from 0 to 28 per 1000 words, even within psychiatrists' own consultations - one psychiatrist recorded a range of 6-32 within four consultations. Moreover, plotting the mean declaratives per 1000 words against adherence (Fig. 1) and therapeutic alliance (Fig. 2) for each psychiatrist showed no apparent clustering effect. However, given that psychiatrists were often involved in a number of patient consultations (a mean of 4.6 per clinician), separate GEE models were fitted to these two outcome variables to account for the potential effect of psychiatrist identity on the data.

\section{Generalised estimating equations}

Each GEE used a gamma distribution with a log link function, and controlled for within-individual correlations of psychiatrist and the three symptom scales using an independent correlation matrix. The independent variables in each case were the proportion of each of the four question types normalised per 1000 words.

\section{Therapeutic alliance}

Even after adjusting for psychiatrist identity and patient symptoms there was a significant main effect on psychiatrists' ratings of the therapeutic alliance in terms of the amount of 'wh-' questions

\begin{tabular}{|c|c|c|c|c|c|c|c|c|}
\hline & \multicolumn{8}{|c|}{ Question type } \\
\hline & \multicolumn{2}{|c|}{ Yes/no } & \multicolumn{2}{|c|}{ 'Wh-' } & \multicolumn{2}{|c|}{ Declarative } & \multicolumn{2}{|c|}{ Tag } \\
\hline & $r$ & $P$ & $r$ & $P$ & $r$ & $P$ & $r$ & $P$ \\
\hline \multicolumn{9}{|l|}{ Symptoms } \\
\hline General & 0.13 & 0.14 & 0.15 & 0.82 & 0.13 & 0.13 & 0.13 & 0.15 \\
\hline PANSS positive & 0.05 & 0.05 & $0.18^{*}$ & 0.04 & 0.03 & 0.75 & 0.14 & 0.10 \\
\hline PANSS negative & $0.18^{*}$ & 0.04 & -0.01 & 0.93 & -0.01 & 0.91 & -0.03 & 0.76 \\
\hline Therapeutic alliance (HAS score) & 0.03 & 0.73 & 0.10 & 0.26 & $0.28^{* *}$ & $<0.01$ & 0.04 & 0.68 \\
\hline Adherence & 0.04 & 0.64 & 0.03 & 0.72 & $-0.20^{\star}$ & 0.02 & 0.14 & 0.13 \\
\hline
\end{tabular}




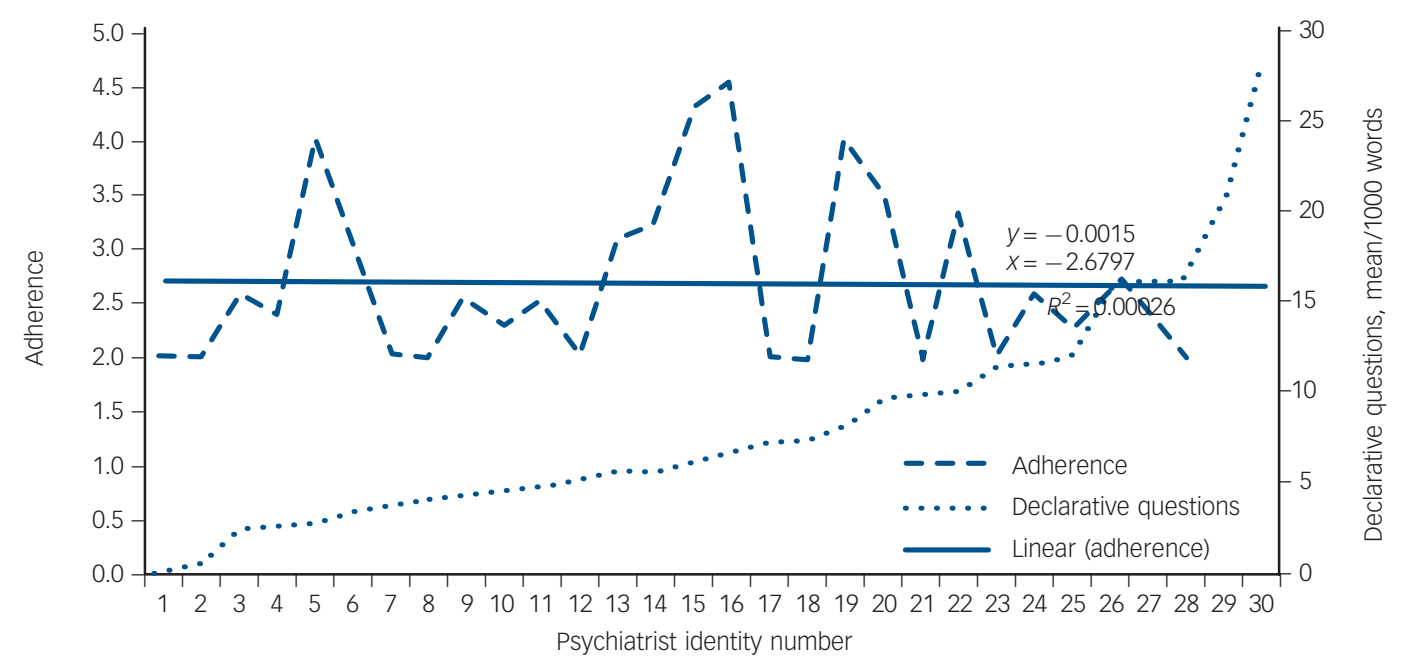

Fig. 1 Adherence and use of declarative questions.

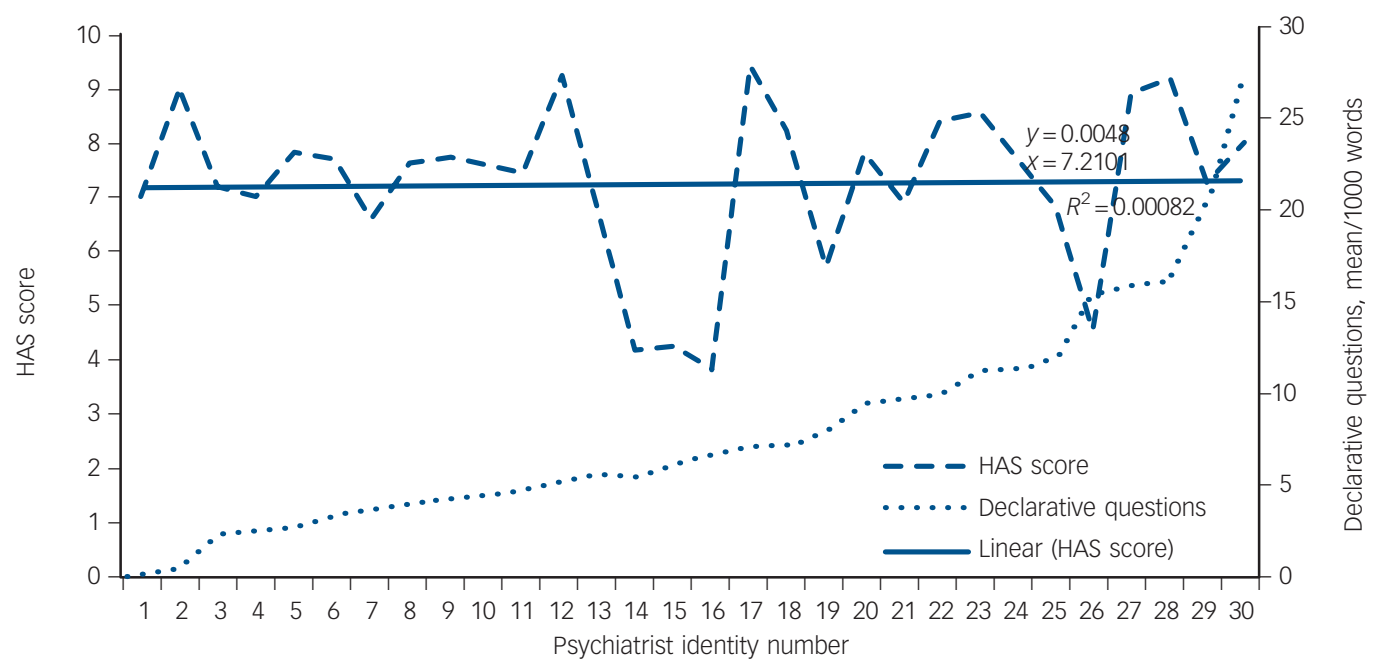

Fig. 2 Therapeutic alliance and use of declarative questions. HAS, Helping Alliance Scale.

and declarative questions that the psychiatrist used, adjusted for the amount of speech (Table 4). However, these effects were in opposite directions: psychiatrists rated the therapeutic alliance as better if they used more declarative questions, and worse if they used more 'wh-' questions.

\section{Adherence}

There was a main effect of declarative questions on adherence, even when controlling for patients' symptoms and the identity of the psychiatrist (Table 4). This suggests that if psychiatrists used more declarative questions in their consultations, patients would be more likely to adhere to their treatment, as measured 6 months after the consultation.

\section{Declarative questions in practice}

Declarative questions were the only question subtype associated with better clinician-rated adherence and therapeutic relationships. This raises the question of what kinds of activity they are performing in practice. On examination of 210 declaratives extracted from a random subset of 30 consultations (with mean frequencies above 3 per 1000 to ensure selected cases contained a sufficient density of questions) three distinctions were immediately observable. A minority appeared in a 'checklist' form (16;8\%) - truncated questions that may represent rapid topic shifts following a patient answer to a prior question, ${ }^{5}$ e.g. 'Sleeping okay?', 'Good appetite?' A slightly larger proportion $(23 ; 11 \%)$ incorporated patients' immediately prior talk, repeating lexical elements verbatim, ${ }^{23}$ for example:

Patient: 'I've had some side-effects.'

Doctor: 'You've had some side-effects?'

The majority of questions, however, displayed a further level of abstraction - conveying 'inferences or assumptions' about the patients' prior talk $(171 ; 81 \%){ }^{5}$ Over half of these were a homogeneous subgroup of 'so-prefaced' inferences (90;53\%) such as, 'So you feel a bit anxious?' (20 specific examples of these questions are given in the Appendix). Each declarative is prefaced by the upshot marker ' $\mathrm{so}^{24}$, following which the psychiatrist invites confirmation of an emotional inference. 'So' indexes inferential or causal connections with the prior talk ${ }^{25}$ and displays 


\begin{tabular}{|c|c|c|c|c|c|c|}
\hline & B & SE & $95 \% \mathrm{Cl}$ & Wald $\chi^{2}$ & d.f. & $P$ \\
\hline \multicolumn{7}{|l|}{ HAS total score ${ }^{a}$} \\
\hline (Intercept) & 1.97 & 0.05 & 1.86 to 2.07 & 1363.58 & 1 & $<0.001$ \\
\hline 'Wh-' questions & -13.74 & 5.41 & -24.34 to -3.13 & 6.44 & 1 & 0.011 \\
\hline Tag questions & 12.74 & 9.11 & -5.13 to 30.60 & 1.95 & 1 & 0.162 \\
\hline Yes/no questions & 0.77 & 3.45 & -6.00 to 7.54 & 0.05 & 1 & 0.824 \\
\hline Declarative questions & 11.60 & 2.27 & 7.15 to 16.04 & 26.14 & 1 & $<0.001$ \\
\hline \multicolumn{7}{|l|}{ Adherence total score ${ }^{\mathrm{b}}$} \\
\hline (Intercept) & 0.95 & 0.09 & 0.77 to 1.13 & 107.90 & 1 & $<0.001$ \\
\hline 'Wh-' questions & 13.36 & 8.52 & -3.34 to 30.05 & 2.46 & 1 & 0.117 \\
\hline Tag questions & 4.82 & 18.33 & -31.12 to 40.75 & 0.07 & 1 & 0.793 \\
\hline Yes/no questions & 3.66 & 6.21 & -8.50 to 15.83 & 0.35 & 1 & 0.555 \\
\hline Declarative questions & -16.40 & 3.43 & -23.12 to -9.68 & 22.89 & 1 & $<0.001$ \\
\hline
\end{tabular}

the psychiatrist working closely with the patient's contribution. In each example the clinician produces a display of understanding, 'formulating' the patient's feelings or perspective, e.g. being 'anxious', 'happy', 'lethargic', 'depressed' or 'under pressure'. Garfinkel \& Sacks ${ }^{26}$ first identified the use of such formulations in interaction:

'a member may treat some part of the conversation as an occasion to describe that conversation, to explain it, or characterise it or explicate, or translate, or summarise or furnish the gist of it . . that is to say, a member may use some part of the conversation as an occasion to formulate the conversation'.26

The formulations in the Appendix characterise the personal salience of the conversation for the patient. The following data fragment shows a 'so-prefaced' declarative question in context: here the psychiatrist edits the patient's talk to highlight its psychological implications:

Patient: It's just that sometimes in the afternoon I get like, you know, I get the feeling that it's going to happen to me, I will end up in the hospital.

Doctor: Okay.

Patient: And, er

Doctor: So you feel a bit anxious?

Patient: Yeah.

Here the psychiatrist uses a declarative question to distil the central theme of a larger stretch of talk concerning the patient's fears about relapse and associated return to an in-patient ward. He proposes - and invites confirmation of - a candidate understanding within an emotional frame of relevance, inferring that the patient's 'feeling that it's going to happen to me' means he is feeling 'anxious'. A similar extract is given below:

Patient: Yeah, I like to chill out in the house, Doctor, you know, I watch telly and then cook something and then washing and tidy the house up, you know?

Doctor: Yeah. So you're quite happy being on your own?

Patient: I'm quite happy, Doctor. Yeah, yeah.

The psychiatrist uses a declarative formulation to propose an understanding of the patient's stance in relation to how he spends his time alone at home. His deduction 'you're quite happy being on your own' is distilled from the patient's 'I like to chill out in the house'. Such formulations have been studied extensively in psychotherapy as devices for suggesting 'something implicitly meant by the client, ${ }^{27}$ which display understanding, cooperation and engagement, yet simultaneously serve clinical objectives. ${ }^{28}$ These intermittent 'summaries' are produced in service of therapeutic interpretation ${ }^{28}$ and, in this context, consistent with a psychiatric point of view. Several implications for understanding psychiatric questioning and the direction of future research can be collectively extracted from these findings.

\section{Discussion}

Psychiatrists can use a range of methods to elicit information from patients by varying the structure of their questions. We captured these alternatives in a coding protocol, usable across a variety of medical contexts. There are three main findings from this study, each with applied significance. Despite the different possibilities of question form, psychiatrists used a relatively small subset frequently: yes/no questions (the prevalence of which is consistent with findings in general medicine), ${ }^{29}$ 'wh-' questions, declarative questions and tag questions. Although this pattern is of interest in its own right, choice between these question types may be consequential for clinical outcomes. Psychiatrists' use of declaratives - statements that invite patient (dis)confirmation (a subclass of 'closed' question) - predicted better psychiatrist perceptions of the therapeutic relationship and subsequent patient adherence at 6 months, after adjusting for symptoms, psychiatrist identity and amount of speech. Conversely, psychiatrist 'wh-' (open) questions, inviting more elaborate responses, correlated with more severe positive symptoms and predicted worse psychiatrist perceptions of the patient relationship. The findings counter commonly held assumptions regarding the conventional binary distinction between open (positive) and closed (negative) questions often used to construe a model of patient-centred care, e.g. using 'open-ended questions to learn about the patient.' Indeed, closer observation of the current data suggests that declarative questions can be deployed to display an understanding of patient experience.

\section{Limitations}

This study should be considered in the context of its limitations. Potential inferences regarding the direction of effect on adherence and the therapeutic relationship are constrained by the statistical methods used here: correlation cannot determine causality. Moreover, encounters included only patients diagnosed with schizophrenia; we cannot with any certainty extrapolate findings to other mental health populations that may have different communicative needs. The construct validity of the outcomes measured should also be considered. Although subjective measures of the therapeutic alliance are well accepted to assess the therapeutic relationship, they are more problematic, albeit heavily relied on, ${ }^{1}$ when assessing adherence. Provider ratings of adherence may be based on the report of the patient or on a worsening clinical condition, which may be related to failure of the chosen medication to control symptoms. ${ }^{18}$ Moreover, doctors' 
ratings of adherence are frequently related to their perception of clinician-patient agreement. ${ }^{31}$ This could go some way towards explaining why alliance and adherence were both associated with declarative questions. The study also does not allow for the fact that patients may have had contacts with other health professionals over the 6-month period. Although it is the psychiatrist with whom the patient makes treatment decisions, other individuals may also have some influence on adherence behaviour.

Our approach to question coding relied on pre-defined properties of a question's form, supporting reliable interrater coding. However, the categories were based predominantly on syntactic structure. This is problematic from some standpoints: what linguistically defines questions as questions does not necessarily define them as interactional objects - a question without the linguistic form of a question may still accomplish questioning, and the form of a question can be used for actions other than questioning. ${ }^{32}$ If there is no exact one-to-one correspondence between form and action, further explanatory potential may lie in contextual qualitative analyses of questions in situ. Our analysis focused on psychiatrists' questions. Previously, we found that the more questions patients asked to clarify the psychiatrist's talk, the more adherent to treatment they were 6 months later. ${ }^{6}$ This raises the question of how psychiatrist questioning affects patient questioning, an avenue for further research.

\section{Clinical implications}

The findings suggest that a more sensitive classification than 'closed' $v$. 'open' is necessary to inform understanding of best questioning practices in psychiatry. Declaratives were the only class of closed question - from six possible subtypes - to be associated with better alliance and adherence. Although these are often labelled as negatively connotated 'leading' questions, ${ }^{4}$ this association (and actual data examples) suggests the function and consequences of declaratives may be more nuanced. Indeed, prior qualitative research of declarative questions in psychotherapy settings aligns with this. By displaying a more 'knowing' stance than other question types, ${ }^{5}$ declaratives create an opportunity for patients to confirm their psychiatrist's grasp of their state of affairs (e.g. 'so you feel a bit anxious?'), such that they can function and be heard as displays of understanding, ${ }^{28}$ empathy, ${ }^{33}$ and active listening. ${ }^{34}$ Arguably, each of these may be be instrumental to the formation of therapeutic rapport and alliance.

Although the objectives and challenges of psychotherapy may be somewhat distinct from psychiatry, this prompts further qualitive research to understand the function of declaratives in psychiatry specifically. In the treatment of schizophrenia, the psychiatrist must balance information gathering with responsivity to patient experience, all the while maintaining an attitude of nonconfrontation and non-collusion. ${ }^{35}$ When proposing how patients might 'feel' on account of their reports, declarative questions may allow clinicians to be sensitive to the emotional aspects of their experiences, while (where appropriate) sustaining a clinically desirable attitude of non- collusion with aspects of content, reconciling these sometimes diametrical requirements. Within the context of reviewing a patient's mental state, interviewing patients without using this kind of device may appear insensitive and be more characteristic of a stilted checklist approach to questioning. It is interesting that this psychotherapeutic practice is associated with better psychiatrist ratings of the therapeutic alliance. Importantly, clinician ratings of the therapeutic alliance have been found to predict outcomes in psychosis, ${ }^{36}$ perhaps reflecting the non-specific factors at play in psychiatry.

The findings here lay out the prospect that training clinicians to ask more declarative questions (or at least certain types) may be one method of improving the therapeutic alliance and subsequent adherence. This hypothesis is based on the direction of effect commonly cited in alliance and adherence research: perceptions of the therapeutic relationship, mediated through talk, may influence adherence. However, given that this particular pathway of causality cannot be confirmed within the scope of a correlational study, an equally interesting alternative is the polar directionality. Through this lens, declaratives represent one possible index for how positive alliances and/or adherence are manifest in interaction (or less favourable alliances, as indexed by 'wh-' questions). The alliance and adherence may be independent variables with discursive consequences: psychiatrists might more easily achieve, display and invite confirmation of their 'understandings' through declarative questions with patients who are more adherent and engaged with treatment in the first place.

Whichever interpretation is used, each highlights the need to consider the degree of shared understanding established in patient-clinician interaction. This is consistent with our earlier study: patient attempts to check understanding (clarifying what the psychiatrist said in a previous turn) were also associated with better adherence. ${ }^{6}$ Relatedly, one would expect achieving mutual understanding might be more difficult in people with symptoms such as delusions. This could explain why 'wh-' questions - open questions that presuppose less understanding, thereby inviting more extensive responses - were associated with symptoms and poorer psychiatrist alliance ratings. Indeed, discussion of psychotic symptoms can cause considerable interactional tension in out-patient encounters. ${ }^{2}$ Recognising candidate interactional markers of good relationships such as declarative questions might be one of the first steps for developing interventions to improve adherence, derived from naturalistic interaction. Crucially, clinician ratings of the therapeutic alliance in psychiatry have been found to predict more distal outcomes. ${ }^{36}$ More abstract notions of 'patient-centredness' do not easily translate into measurable communication practices, conducive to training and research.

This study underlines the need for specificity and presents a candidate questioning practice for further analysis. Psychotic symptoms are associated with increased risk of suicide, ${ }^{37}$ and treatment non-adherence accounts for approximately $40 \%$ of readmission to hospital in the 2 years following discharge from in-patient treatment in schizophrenia, ${ }^{38}$ incurring substantial clinical and economic burdens. Given that the ultimate goals of interaction in psychiatric settings are the amelioration of such symptoms and the prevention of relapse, the stakes involved in empirically grounded 'good' questioning are very high indeed.

\footnotetext{
Laura Thompson, PhD, Department of Social Sciences, Loughborough University, Loughborough, UK; Christine Howes, PhD, Department of Philosophy, Linguistics and Theory of Science, University of Gothenburg, Sweden; Rose McCabe, PhD, University of Exeter Medical School, Exeter, UK

Correspondence: Dr Laura Thompson, Department of Social Sciences, Loughborough University, Leicestershire LE11 3TU, UK. Email: I.thompson2@ Iboro.ac.uk

First received 22 Apr 2014, final revision 14 Mar 2015, accepted 18 Mar 2015
}

\section{Funding}

The research was supported by funding from the UK Medical Research Council (G0401323).

\section{Acknowledgements}

The authors gratefully acknowledge the work of Dr Mary Lavelle and Dr Nadia Strapelli who contributed to the piloting of the coding protocol and/or final coding. The input of Professor contributed to the piloting of the coding protocol and/or final coding. The input of Professor
Paul Drew and Dr Suzanne Beeke, who commented on an earlier version of this work, is also greatly appreciated. 


\section{Appendix}

\section{Psychiatrists' 'so-prefaced' declarative questions}

So you are feeling not so well?

So you feel a bit anxious?

So you're quite happy being on your own?

So you're lethargic, you just couldn't be bothered to do these things?

So you feel okay about it?

So that's something you want to switch off from?

So you are quite happy to continue with the risperidone?

So you're under a lot of pressure at the moment?

So you got a little bit depressed?

So you feel anxious about the amount you're eating?

So you have episodes when you feel really bad?

So you think you're better off?

So you're feeling better in any case?

So the things that you find difficult now are your self-confidence?

So but overall you feel better in yourself?

So I think in terms of what we're doing at the moment you are quite satisfied?

So you're not feeling well?

So these have been helpful?

So on the whole from a psychiatric point of view you're very stable?

So you'd be worried about the antidepressant?

\section{References}

1 Thompson L, Mccabe R. The effect of clinician-patient alliance and communication on treatment adherence in mental health care: a systematic review. BMC PSychiatry 2012; 12: 87.

2 McCabe R, Heath C, Burns T, Priebe S. Engagement of patients with psychosis in the consultation: a conversation analytic study. BMJ 2002; 325 1148-51.

3 Priebe S, Dimic S, Wildgrube C, Jankovic J, Cushing A, McCabe R. Good communication in psychiatry: a conceptual review. Eur Psychiatry 2011; 7 403-7.

4 Burton N. Psychiatry. Wiley Blackwell, 2010.

5 Heritage J. Questioning in medicine. In Why Do You Ask? The Function of Questions in Institutional Discourse (eds AF Freed, S Ehrlich): 42-68. OUP, 2010

6 McCabe R, Healey P, Priebe S, Bremner S, Lavelle M, Dodwell D, et al. Shared understanding in psychiatrist-patient communication: association with treatment adherence in schizophrenia. Patient Educ Couns 2013; 93: 73-9.

7 American Psychiatric Association. Diagnostic and Statistical Manual of Mental Disorders (4th edn, text revision) (DSM-IV-TR). APA, 2000.

8 Groenendijk J, Stokhof M. Questions. In Handbook of Logic and Language (eds J van Benthem, A ter Meulen): 1055-124. MIT Press, 1997.

9 Ginzburg J, Sag IA. Interrogative Investigations. CLSI, 2001.

10 Drew P. 'Open' class repair initiators in response to sequential sources of trouble in conversation. Lang Cogn Process 1997; 2: 19-41.

11 Heritage J, Clayman S. Talk in Action: Interactions, Identities and Institutions Wiley Blackwell, 2010.

12 Stivers T, Rossano RF. Mobilising response. Res Lang Soc Interact 2010; 43 3-31.

13 Safarova M, Swerts $M$. On recognition of declarative questions in English. In Proceedings of Speech Prosody 2004 Conference: 313-6. Nara, 2004. Available at: http://www.cnts.ua.ac.be/papers/2004/spn04.pdf.
14 Raymond G. Grammar and social relations: alternative forms of yes/no type initiating actions in health visitor interaction. In Why Do You Ask? The Function of Questions in Institutional Discourse (eds AF Freed, S Ehrlich): 87-107. OUP, 2010.

15 Dexter Coder. Dexter: Tools for Analysing Language Data. Dexter Coder, 2011 (http://www.dextercoder.org)

16 Kay S, Fiszbein A, Opler L. The positive and negative syndrome scale (PANSS) for schizophrenia. Schizophr Bull 1987; 13: 261-76.

17 Priebe S, Gruyters T. The role of the helping alliance in psychiatric community care: a prospective study. J Nerv Ment Dis 1993; 181: 552-7.

18 Velligan DI, Lam YWF, Glahn DC, Barrett JA, Maples NJ, Ereshefsky L, et al. Defining and assessing adherence to oral anti-psychotics: a review of the literature. Schizophr Bull 2006; 32: 724-42.

19 Buchanan A. A two-year prospective study of treatment compliance in patients with schizophrenia. Psychol Med 1992; 22: 787-97.

20 SPSS. PASW Statistics 18.0. SPSS, 2009.

21 Liang $K Y$, Zeger SL. Longitudinal data analysis using generalised linear models. Biometrika 1986; 73: 13-22.

22 Ballinger GA. Using generalized estimating equations for longitudinal data analysis. Organ Res Methods 2004; 7: 127-50.

23 Robinson JD, Kevoe-Feldman H. Using full repeats to initiate repair on others' questions. Res Lang Soc Interact 2010; 43: 232-59.

24 Schiffrin D. Discourse Markers. CUP, 1987.

25 Bolden G. Implementing incipient actions: the discourse marker 'so' in English converstion. J Pragmat 2009; 45: 974-98.

26 Garfinkel S, Sacks H. On formal structures of practical actions. In Theoretical Sociology (eds JC McKinney, EA Tiryakian): 337-66. Appleton-Century-Crofts, 1970.

27 Bercelli F, Rossano F, Viaro M. Clients' responses to therapists reinterpretations. In Conversation Analysis and Psychotherapy (eds A Perakyla, C Antaki, S Vehvilainen, I Leudar): 43-61. CUP, 2008.

28 Antaki C. Formulations in psychotherapy. In Conversation Analysis of Psychotherapy (eds A Perakyla, C Antaki, S Vehvilainen, I Leudar): 26-42. CUP, 2008.

29 Roter DL, Hall JA. Health education theory: an application to the process of patient-provider communication. Health Educ Res 1991; 6: 185-93.

30 Hanyok LA, Hellman DB, Rand C, Zeigelstein RC. Practicing patient centred care: the questions clinically excellent physicians use to get to know their patients as individuals. Patient 2012; 5: 141-5.

31 Phillips LA, Leventhal EA, Leventhal $\mathrm{H}$. Factors associated with the accuracy of physicians' predictions of patient adherence. Patient Educ Couns 2011; 85: 461-7.

32 Schegloff EA. On some questions and ambiguities in conversation. In Structures of Social Action (eds J Maxwell Atkinson, J Heritage): 28-53. CUP, 1984.

33 Ruusuvuori J. Empathy and sympathy in action: attending to patients' troubles in Finnish homeopathic and general practice consultations. Soc Psychol Q 2005; 68: 204-22.

34 Hutchby I. Active listening: formulations and the elicitation of feeling-talk in child counseling. Res Lang Soc Interact 2005; 38: 303-29.

35 Turkington D, Siddle R. Cognitive therapy for the treatment of delusions. Adv Psychiatr Treat 1998; 4: 235-42.

36 Priebe S, Richardson M, Cooney M, Oluwatoyin A, McCabe R. Does the therapeutic relationship predict outcomes of psychiatric treatment in patients with psychosis? A systematic review. Psychother Psychosomat 2011; 80: 70-7.

37 Palmer BA, Pankratz S, Bostwick JM. The lifetime risk of suicide in schizophrenia. Arch Gen Psychiatry 2005; 62: 247-53.

38 Weiden P, Olfson M. Cost of relapse in schizophrenia. Schizophr Bull 1995; 21: $419-29$ 